\title{
Impact of Precautionary Lockdown Measures During the COVID-19 Pandemic on the Development of Digital Eye Strain Among Contact Lens Users
}

\author{
Balsam Alabdulkader \\ Optometry and Vision Sciences, College of Applied Medical Sciences, King Saud University, Riyadh, Saudi Arabia \\ Email address: \\ balabdulkader@ksu.edu.sa \\ To cite this article: \\ Balsam Alabdulkader. Impact of Precautionary Lockdown Measures During the COVID-19 Pandemic on the Development of Digital Eye \\ Strain Among Contact Lens Users. International Journal of Ophthalmology \& Visual Science. Vol. 6, No. 2, 2021, pp. 94-100. \\ doi: $10.11648 /$ j.ijovs.20210602.16
}

Received: April 13, 2021; Accepted: April 24, 2021; Published: April 29, 2021

\begin{abstract}
Strict lockdown measures were implemented to prevent the spread of COVID-19, which increased the reliance on digital electronic devices in the performance of daily living activities. The extensive use of digital devices was associated with the development of digital eye strain (DES) symptoms, which is more prevalent in contact lens (CL) wearers. This study aimed to investigate the number of hours spent on digital devices and the incidence of associated symptoms in CL wearers during a 24-h lockdown. This cross-sectional study used a self-reported questionnaire to obtain the following data: a) demographic information, b) CL wear profile, c) CL wear during the lockdown, d) total number of hours spent on digital devices, and e) 15 DES-related symptoms. A total of 82 CL wearers $(29.6 \pm 10.8$ years; $88 \%$, female $)$ were recruited. The median total number of hours spent on digital devices during the lockdown was 10 . The number of hours spent on smartphones significantly increased during the lockdown compared to that before the lockdown (5.5 VS 7 hours; $p=0.000)$. DES was reported in $83 \%$ of participants. The most common reported symptoms were eye strain $(50 \%)$, dryness (48\%), and headache (40\%). The majority of participants (56\%) continued wearing their CL during the lockdown. The incidence of DES was significantly higher in participants who were highly engaged with digital devices compared to the less engaged participants during the lockdown $(\mathrm{p}=0.001)$. Prolonged use of digital devices increases the risk of DES-related symptoms in CL wearers. Intervention strategies for CL wearers may be important in optimizing the use of digital devices and decreasing the risk of DES-related symptoms.
\end{abstract}

Keywords: Digital Eye Strain, COVID-19, Contact Lenses, Computer Vision Syndrome, Smartphones, Lens Wear

\section{Introduction}

In March 2020, the World Health Organization announced the outbreak of coronavirus disease 2019 (COVID-19) as a global pandemic, which first reported at the end of 2019 in Wuhan, China. The pathogen of COVID-19 is a new member of the Coronaviridae family, severe acute respiratory syndrome coronavirus 2 (SARS-CoV-2) [1], which is believed to be transmitted through respiratory droplets [2]. The infection could be transferred by direct contact with patients with COVID-19 or indirectly - by contact with contaminated objects followed by touching the face and eyes [2]. The pandemic forced many countries to implement strict rules to prevent the spread of the virus. These measures included flight suspension, lockdown enforcement, distance learning implementation, and work-from-home regulations. Hence, the motto of the COVID-19 period was "Stay at Home." A frequently used phrase to encourage individuals to stay home to practice social distancing and limit outdoor activities to the essential needs (i.e., groceries and pharmacies). Similar to many governments, the Saudi Arabian government implemented extreme precautionary measures to mitigate the rapid spread of the disease. A nationwide partial lockdown was established and then tightened to a 24-h lockdown across the kingdom, which 
lasted 91 and 21 days, respectively.

Home quarantine caused individuals to rely on their electronic digital devices to accomplish work and school tasks and entertainment activities. Electronic digital devices, such as televisions, smartphones, tablets, and computers, play a major role in our modern society. As a result of the mandatory indoor living, individuals were more reliant on electronic devices for study, work, and nonvocational activities. Therefore, the tendency to spend more time using digital devices is expected to increase during the lockdown. Indoor activities are arguably limited to short distances compared to outdoors. Hence, the extensive increase in the use of digital devices may have caused individuals to develop ocular discomfort.

Digital eye strain (DES) is characterized by several ocular and visual symptoms resulting from prolonged use of electronic devices [3]. The most common symptoms are eye strain, blurred vision, headache, and dry eyes. The American Optometric Association recommends several solutions to alleviate DES-related symptoms, including (1) following the 20-20-20 rule, which is resting for $20 \mathrm{~s}$ by viewing a target $20 \mathrm{ft}$ away every $20 \mathrm{~min}$ [3], (2) use of lenses that meet the required visual demand, and (3) proper body positioning for computer use [3]. Moreover, DES-related symptoms are not limited to the eyes and vision. They include symptoms on other body parts, such as neck and shoulder pain. Several studies reported that the prevalence of DES ranges from 50\% to $90 \%$ among different populations [4-8] and is higher among contact lens wearers compared to non-contact lens wearers $[9,10,6,11]$. The proportion of contact lens wearers in Saudi Arabia varies in the current literature from $40 \%$ to $70 \%[12,13]$. As evidence suggests the negative effects of DES on contact lens wearers, there is a need to understand the possible impact of the precautionary measures of the COVID-19 pandemic on the development of DES in contact lens wearers. This study aimed to assess changes in the number of hours of digital devices use during the lockdown and investigate DES-related symptoms in contact lens wearers.

\section{Methods}

\subsection{Setting}

This study adopted an observational, cross-sectional design and was conducted in Saudi Arabia during the 24-h lockdown due to the COVID-19 pandemic. Adult contact lens wearers who were aged $>18$ years and residents of Saudi Arabia who were present during the 24-h lockdown were included in the study. The snowball sampling method was used in the recruitment. Participants were contacted via social media (Twitter, LinkedIn, and WhatsApp) using an online questionnaire that was prepared using Google Forms.

\subsection{Data Collection}

A self-administered online questionnaire was used to collect the data. The questionnaire was divided into five parts:
(1) demographic information and questions on the types of digital devices, use of lubricating drops, and awareness and practice of the 20-20-20 rule; (2) contact lens type and replacement frequency, continuation of contact lens use during the lockdown, and reasons for discontinuation; (3) time spent on using digital devices; (4) scheduled breaks during computer use and their frequency and length; and (5) 15 DES-related symptoms $[4,5,14,6,8]$. The duration of digital device use was assessed by asking participants to access the screen-time feature on their smartphones and report the average daily hours of use during and before the lockdown period. They were also asked to estimate the number of hours spent using the computer. Finally, they were asked to report the total number of hours spent on using digital devices on a daily basis.

DES-related symptoms were assessed by asking contact lens wearers to report if they feel any of these symptoms during the lockdown compared to before the lockdown. The answers followed a 4-point Likert scale ranging from not applicable, less than before, same as before, to more than before. DES was confirmed for study participants who complained of at least one or more DES-related symptoms during the lockdown compared to that before the lockdown. Twenty volunteers piloted the questionnaire before it was dismissed to the public. Volunteers' responses were excluded from the final sample. The time required to complete the questionnaire was approximately 10 minutes. The questionnaire design accepted only complete responses, which made the risk of missing data minimal. However, responses that had clear logical errors or duplicates were excluded from the final sample. This study followed the tenets of the Declaration of Helsinki and was approved by the Ethics Committee of King Saud University (E-20-4802). Informed consent was obtained electronically from all participants before the study enrolment.

\subsection{Statistical Analysis}

Descriptive analysis was performed, and data were presented as frequencies and percentages (\%) for categorical variables. Normality was tested using the Kolmogorov-Smirnov test. The Wilcoxon signed-rank test was performed to interpret the difference in the hours of smartphone use before and during the lockdown. The engagement level with digital devices was classified into two groups: (1) highly-engaged for participants who reported being employed or students, (2) less-engaged for participants who reported being unemployed. Chi-squared test were used to investigate the association between the engagement level and the incidence of DES. The incidence of DES was considered if at least one or more symptoms were reported. Statistical analysis was performed using SPSS 27 (IBM, Chicago, IL, USA), and graphs were generated using GraphPad Prism 8.4.3 (GraphPad Software, San Diego, USA). A P-value $<0.05$ indicated statistical significance. 


\section{Results}

\subsection{Participants' Sociodemographics}

The study included 82 contact lens wearers, of which $88 \%$ were female. The age ranged from 18 to 60 years. In terms of education, the study sample consisted of high schoolers $(n=12$ $14 \%)$, diploma holders $(n=6 ; 7 \%)$, graduates $(n=48 ; 59 \%)$, and postgraduates $(n=16 ; 20 \%)$, of which only $15 \%(n=12)$ were unemployed. Participants' sociodemographic data are presented in Table 1. Interestingly, only 24 participants were aware of the 20-20-20 rule, of which five participants reported frequently practicing it, whereas 15 participants reported occasionally practicing it.

Table 1. Participants'sociodemographic characteristics.

\begin{tabular}{|c|c|}
\hline $\begin{array}{l}\text { Mean age in years }(\mathrm{SD}) \\
\text { Sex }(\%)\end{array}$ & $29.6(10.8)$ \\
\hline Female & $72(88)$ \\
\hline $\begin{array}{l}\text { Male } \\
\text { Education (\%) }\end{array}$ & $10(12)$ \\
\hline Postgraduate & $16(20)$ \\
\hline Graduate & $48(59)$ \\
\hline Diploma & $6(7)$ \\
\hline $\begin{array}{l}\text { High school } \\
\text { Employment status (\%) }\end{array}$ & $12(14)$ \\
\hline Employed/student & $70(85)$ \\
\hline $\begin{array}{l}\text { Unemployed } \\
\text { Use of lubricating drops }\end{array}$ & $12(15)$ \\
\hline Yes & $53(65)$ \\
\hline No & $29(35)$ \\
\hline $20-20-20$ rule awareness & \\
\hline Yes & $24(29)$ \\
\hline No & $58(71)$ \\
\hline $20-20-20$ practice $(\%)$ & \\
\hline Yes & $5(21)$ \\
\hline No & $4(17)$ \\
\hline Sometimes & $15(62)$ \\
\hline Mean age in years (SD) & $29.6(10.8)$ \\
\hline $\operatorname{Sex}(\%)$ & \\
\hline Female & $72(88)$ \\
\hline Male & $10(12)$ \\
\hline Education (\%) & \\
\hline Postgraduate & $16(20)$ \\
\hline Graduate & $48(59)$ \\
\hline Diploma & $6(7)$ \\
\hline High school & $12(14)$ \\
\hline
\end{tabular}

\subsection{Digital Device Use}

The majority of study participants used two or more digital devices $(n=59 ; 72 \%)$ on a daily basis. The devices most commonly used by the participants were smartphones $(n=82$, $100 \%)$, followed by computers $(n=47,57 \%)$ and televisions $(n=29 ; 35 \%)$, whereas the least commonly used devices were videogame consoles $(n=12 ; 15 \%)$ and tablets $(n=10 ; 12 \%)$ (Table 2). The number of hours of digital devices use was not normally distributed $(\mathrm{p}>0.05)$ (Table 2$)$, and nonparametric tests were employed. There was a significant increase in the number of hours of smartphone use before and during the lockdown $(\mathrm{p}=0.000)$ (Table 2$)$.
Table 2. Participants' digital device use.

\begin{tabular}{ll}
\hline Median smartphone hours before lockdown (IQR) & $5.5(4)$ \\
Median smartphone hours during lockdown (IQR) & $7(5)$ \\
Median all digital device hours during lockdown (IQR) & $10(7.25)$ \\
Use of digital devices (\%) & \\
Smartphone & $82(100)$ \\
Computer & $47(57)$ \\
TV & $29(35)$ \\
Videogame consoles & $12(15)$ \\
Tablet & $10(12)$ \\
Number of digital devices used (\%) & \\
1 & $23(28)$ \\
2 & $35(43)$ \\
3 & $13(16)$ \\
4 & $10(12)$ \\
5 & $1(1)$ \\
\hline
\end{tabular}

The use of a computer for work purposes was reported by $73 \%(60 / 82)$ of participants. The median number of hours of computer use during the lockdown was $6 \mathrm{~h}$ and ranged from 1 to $20 \mathrm{~h}$ daily. The majority (67\%) of computer users stated that they do take breaks while working; however, (22\%) revealed that these breaks were taken after $>60 \mathrm{~min}$ of continuous work. Table 3 shows the characteristics of computer use.

Table 3. Computer use and break profiles.

\begin{tabular}{ll}
\hline Median computer hours (range) & $6(1-20)$ \\
Use of computers for work (\%) & \\
Yes & $60(73)$ \\
No & $10(12)$ \\
Computer breaks $(\%)$ & \\
Yes & $55(67)$ \\
No & $15(18)$ \\
Break frequency $(\%)$ & \\
Every 15 min & $11(13)$ \\
Every 20 min & $8(10)$ \\
Every 30 min & $8(10)$ \\
Every 60 min & $10(12)$ \\
Every $>60$ min & $18(22)$ \\
Median break length in minutes (range) & $15(1-60)$ \\
\hline
\end{tabular}

\subsection{Contact Lens Wear Profile}

Of the 82 participants, $68(82.9 \%)$ and $3(3.7 \%)$ were soft contact lens and rigid gas-permeable contact lens users, respectively. Only one participant used scleral contact lenses $(1.2 \%)$, and the remaining participants $(n=10 ; 12.2 \%)$ used other types.

The frequency of replacements of the contact lenses worn by participants widely varied. The majority of participants wore one-day disposables and one-month replacement soft contact lenses (24/82 [29\%] and 22/82 [27\%], respectively). The complete results of contact lens replacement frequencies are shown in Figure 1.

Less than half of the study participants $(n=36 ; 44 \%)$ reported that there was a reduction in contact lens wear during the lockdown. The most common attributes of that reduction are presented in Figure 2. 




Figure 1. Contact lens replacement frequencies.

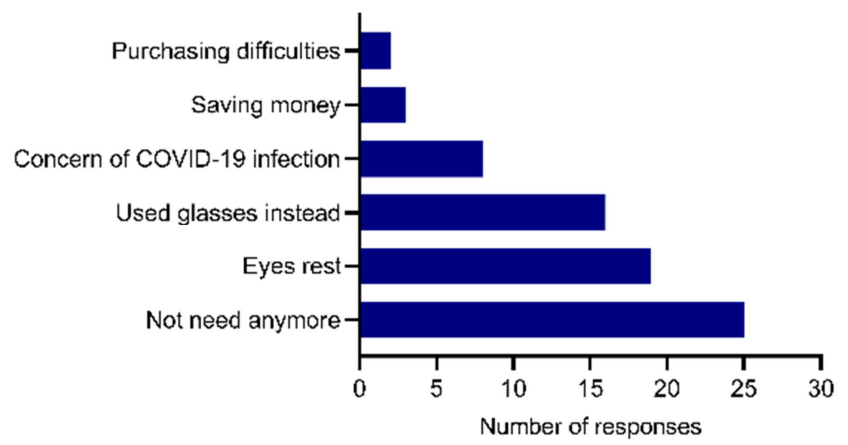

Figure 2. Reasons for cessation of contact lens use during the lockdown.

\subsection{DES-related Symptoms}

The incidence of DES in the study participants was $83 \%$. Among the 15 DES-related symptoms included in the survey, eye strain $(50 \%)$, dryness $(48 \%)$, and headache $(40 \%)$ were the most common symptoms reported during the lockdown. In contrast, the least common DES-related symptoms were double vision (11\%) and foreign body sensation (12\%). Participants' responses to all investigated DES-related symptoms are shown in Table 4.

A subsequent analysis was conducted to investigate the incidence of DES in participants who reported that they continued wearing their contact lenses compared to participants who ceased contact lens wear during the lockdown period ( $87 \%$ vs $77.8 \%$, respectively). The response for self-reported symptoms of participants who continued to wear their contact lenses during the lockdown was similar to the whole group with the top three reported symptoms eye strain (57\%), dryness (54\%), and headache (48\%).

The association between the engagement level with digital devices and DES-incidence was conducted by chi-squared tests. The highly and less engaged groups had $91 \%$ vs $9 \%$ incidence of DES, respectively. The high-engagement with digital devices was significantly associated with the incidence of DES for contact lens wearers during the lockdown period $(\mathrm{p}=0.001)$.

Table 4. Responses of DES-related self-reported symptoms during the 24-h lockdown $(n=82)$.

\begin{tabular}{|c|c|c|c|c|}
\hline DES symptoms & $\mathbf{N} / \mathbf{A}$ & Less than before & Same as before & More than before \\
\hline Eye strain & $20 \%$ & $6 \%$ & $24 \%$ & $50 \%$ \\
\hline Dryness & $21 \%$ & $2 \%$ & $29 \%$ & $48 \%$ \\
\hline Headache & $27 \%$ & $11 \%$ & $22 \%$ & $40 \%$ \\
\hline Excessive blinking & $38 \%$ & $0 \%$ & $27 \%$ & $35 \%$ \\
\hline Itchiness & $34 \%$ & $10 \%$ & $24 \%$ & $32 \%$ \\
\hline Blurred vision & $54 \%$ & $2 \%$ & $16 \%$ & $28 \%$ \\
\hline Burning sensation & $43 \%$ & $4 \%$ & $27 \%$ & $27 \%$ \\
\hline Tearing & $46 \%$ & $11 \%$ & $22 \%$ & $21 \%$ \\
\hline Heavy eyelids & $49 \%$ & $5 \%$ & $27 \%$ & $20 \%$ \\
\hline Sensitivity to bright light & $48 \%$ & $4 \%$ & $30 \%$ & $18 \%$ \\
\hline Eye redness & $51 \%$ & $5 \%$ & $30 \%$ & $13 \%$ \\
\hline Eye pain & $59 \%$ & $4 \%$ & $26 \%$ & $12 \%$ \\
\hline Foreign body sensation & $72 \%$ & $0 \%$ & $16 \%$ & $12 \%$ \\
\hline
\end{tabular}

\section{Discussion}

The COVID-19 pandemic forced societies to make significant adjustments to their daily routines. Strict lockdown rules substituted work, school, and entertaining face-to-face social interactions with virtual ones, which necessitate the use of digital devices. The results of the current study indicated that the use of digital devices significantly increased during the lockdown, where most contact lens wearers develop DES-related symptoms.

The median number of hours of smartphone use significantly increased during the lockdown to that before the lockdown (7 vs 5.5). The median number of hours spent on all devices during the lockdown was 10 . Similarly, a recent study revealed that the average use of digital devices during a lockdown in Saudi was 10.2 h [15]. The level of engagement with digital devices was associated with the incidence of DES. Most study participants (85\%) were employed or students. Therefore, they were more prone to using digital devices to perform work-related tasks. The vast majority of participants used two or more digital devices on a daily basis; the use of more than one device has been reported to increase the risk of DES-related symptoms $[16,17]$.

It is worth noting that the median number of hours spent on smartphones $(7 \mathrm{~h})$ was more than the median time spent on computers $(6 \mathrm{~h})$ and more than half of the total number of hours spent on all devices $(10 \mathrm{~h})$. The observed increase in hours of smartphone use could be attributed to their ease of use and increased access to social media and/or communication with friends/family. Additionally, individual preferences play a major role in the selection of devices, whereby the majority of virtual educational platforms and professional meeting applications can be accessed using smartphones. 
The pandemic's lockdown resulted in intensive use of digital devices, and as a result, DES was reported by $83 \%$ of contact lens wearers. Several studies have associated DES with prolonged use of digital devices [15, 18-20], and some studies revealed a greater impact on contact lens wearers compared to non-contact lens wearers [6, 21-24]. Eye strain and dryness were the most common symptoms in contact lens wearers during the lockdown period $(50 \%$ and $48 \%$, respectively). These results are similar to those of other studies that evaluated contact lens wearers' DES-related self-reported symptoms and use of digital devices. Eye strain is the most common symptom in other studies [15, 19, 25, 26]. Tauste et al. [6] compared DES-related symptoms in contact lens wearers and non-contact lens wearers and reported that the most common symptom was dryness, which was reported by $73 \%$ of contact lens wearers, compared to $36 \%$ of non-wearers. Moreover, Uchino et al. [22, 24] investigated dry eye disease (DED) in the Japanese population and assessed several factors that are associated with DED. The results of their studies showed that contact lens wearers were at a higher risk of developing DED compared to non-contact lens wearers and observed a higher prevalence of DED in contact lens wearers who use digital devices than non-contact lens wearers [22]. Dry eye and visual symptoms increase with the duration of computer use in contact lens wearers compared to non-wearers, where environmental factors trigger these symptoms, as reported by Kojima et al. [23]. Dryness is a major contributor to DES [27, 22, 23] and may be more prevalent and aggravated with environmental conditions, such as climate temperatures and humidity levels, use of air-conditioned environments, and exposure to smoke and dust $[21,23,27,28]$. The extremely hot, dry climate (in most regions) in Saudi Arabia necessitates the use of air conditioners, where studies have shown that these factors contribute to the development of dry eyes, especially in contact lens wearers $[21,29,30]$. The remaining symptoms have been reported in different percentages across different studies $[6,21]$. This might be due to the variant methods, samples, and objectives between studies. However, these comparisons should be interpreted with caution because of the different questionnaires used across studies. Furthermore, this study was conducted at a unique time (24-h lockdown), which may not be easy to replicate, explaining the high use of digital devices and increase in DES-related symptom complaints compared to those in previous studies.

Although the hours were extended to $20 \mathrm{~h}$ /day for some participants, yet only about a third of the study participants $(n=24)$ were aware of the 20-20-20 rule, of which only five participants reported practicing it. Only $10 \%$ of participants who used computers took a break every $20 \mathrm{~min}$, while $44 \%$ reported that they took a break every hour or more. Frequent breaks assist in relaxing the accommodative system, which in turn reduces DES-related symptoms [31, 27]. Public education is needed on the consequences of infrequent breaks during digital device use. Effective patient education strategies provided by eye care practitioners to contact lens wearers should emphasize the importance of practicing the
20-20-20 rule and the consequences of eye exhaustion. Educational materials should be offered in the patients' preferred format (verbal, pamphlet with illustrations, website, or video). Eye care practitioners should provide verbal education on the importance of scheduled breaks at every eye appointment.

The prolonged use of digital devices reduces the blink rate, which can lead to dry eyes [27, 32, 33]. Artificial tears were recommended to relieve dryness symptoms and aid in eye lubrication $[31,34]$. It was determined that the use of artificial tears could help decrease [31, 35], but not resolve [34], ocular discomfort associated with digital device use. Although the majority of participants $(65 \%)$ reported using lubricating drops, the rate of DES-related symptoms was still high. However, with extensive digital device use, DES-related symptoms may surpass the comforting effect of the lubricating drops.

Reduced contact lens wear during the pandemic's lockdown was reported by $44 \%$ of the participants. These results are similar to those of recent studies conducted in the UK and Spain, which reported a decrease in contact lens wear during the COVID-19 pandemic ( $72 \%$ and $46 \%$, respectively) [36, 37]. In this study, the most common reason attributed to decreased contact lens wear was the limited need for them while staying at home $(30 \%)$, which is consistent with the results of similar studies [36, 37]. The reduced need for contact lens wear during the lockdown could be because of the desire for a better appearance while socializing or participating in active sports, which were not possible during the lockdown. In contrast, $10 \%$ reported contact lens wear reduction due to fear of COVID-19. This fear may arise from the recommendations and awareness campaigns to avoid touching the face and eyes. It is essential to mention that, with the currently provided evidence, there is no association found between contact lens wear and risk of COVID-19 infection [38]. However, several studies have reported the presence of ocular manifestation during the course of COVID-19, which cannot be ignored $[39,40]$. Therefore, eye care practitioners must assure contact lens wearers to comply with their contact lens hygiene protocols, including hand washing and adherence to contact lens replacement times, and cease the use of contact lenses and consult their physicians when suspecting any ocular infections [38]. Other reasons for contact lens wear reduction during the lockdown were as follows: giving the eyes a break (32\%), using glasses instead (20\%), saving money (4\%), and purchasing difficulties (3\%).

The study limitations include the reliance on a self-reported questionnaire. However, the questionnaire approach and lack of objective measurements were a result of the enforcement of a 24-h lockdown rule in Saudi Arabia. Moreover, the duration of the cessation of contact lens wear during the lockdown was not investigated, as it was not clear how long the lockdown will last. This suggests that the cessation period may vary between participants in that group. Furthermore, some participants may have exaggerated the reporting of certain symptoms and biased toward psychological effects due to the lockdown. A notable strength of the study is the use of the 
"screen-time" feature in smartphones to measure the time spent on using smartphones, which we believe to be more accurate than participants' estimates, which are recalled, or records from diaries.

Eye care providers may have observed the increase in DES cases than before the pandemic. To alleviate DES-related symptoms, the American Optometric Association [4] recommended several solutions to alleviate DES-related symptoms, which include the following: 1) regular eye examinations, 2) use of lenses that meet special visual demands (e.g., computer glasses), and 3) environmental and behavioral adjustments while using digital devices, such as a proper distance from the screen, appropriate lighting, and frequent breaks.

\section{Conclusion}

The present study revealed that, during the 24-h lockdown, the hours spent on using digital devices significantly increased for contact lens wearers. As a result, contact lens wearers developed ocular and visual symptoms that are associated with DES. The majority of participants demonstrate a low level of knowledge of the 20-20-20 rule. The provision of information and effective educational strategies should be developed to increase awareness of the importance of regular eye examinations and following the 20-20-20 rule to help reduce DES-related symptoms.

\section{Conflict of Interest}

All the authors do not have any possible conflicts of interest.

\section{Acknowledgements}

The author thanks Ms. Suhaliah Alamri for her assistance and support during the preparation of this manuscript. The author also thank the Deanship of Scientific Research and RSSU at King Saud University for their technical support.

\section{References}

[1] Lu R, Zhao X, Li J, et al. Genomic characterisation and epidemiology of 2019 novel coronavirus: implications for virus origins and receptor binding. The Lancet. 2020.

[2] World Health Organization: Coronavirus disease 2019 (COVID-19). Situation Report - 73. World Health Organization 2020, (March):2633.

[3] American Optometric Association. Computer vision syndrome. American Optometric Association. [cited 2020 June 29]. Available

at:https://www.aoa.org/patients-and-public/caring-for-your-vis ion/protecting-yourvision/computer-vision-syndrome

[4] Hayes J, Sheedy J, Stelmack J, et al. Computer use, symptoms, and quality of life. Optom Vis Sci. 2007; 84 (8): 739-45.

[5] Portello JK, Rosenfield M, Bababekova Y, et al.
Computer-related visual symptoms in office workers. Ophthalmic Physiol Opt. 2012; 32 (5): 375-82.

[6] Tauste A, Ronda E, Molina MJ, et al. Effect of contact lens use on Computer Vision Syndrome. Ophthalmic Physiol Opt. 2016; 36: 112-19.

[7] Coles-Brennan C, Sulley A, Young G. Management of digital eye strain. Clin Exp Optom. 2019; 102 (1): 18-29.

[8] Xu Y, Deng G, Wang W, et al. Correlation between handheld digital device use and asthenopia in Chinese college students: a Shanghai study. Acta Ophthalmol (Copenh). 2019; 97 (3): e442-e47.

[9] Wiggins NP, Daum KM, Snyder CA. Effects of residual astigmatism in contact lens wear on visual discomfort in VDT use. J Am Optom Assoc. 1992; 63 (3): 177.

[10] Shimmura S, Shimazaki J, Tsubota K. Results of a population-based questionnaire on the symptoms and lifestyles associated with dry eye. Cornea. 1999; 18 (4): 408-11.

[11] Tauste A, Ronda E, Molina MJ, et al. Effect of contact lens use on Computer Vision Syndrome. Ophthalmic Physiol Opt. 2016; 36: 112-16.

[12] Abahussin M, AlAnazi M, Ogbuehi KC, et al. Prevalence, use and sale of contact lenses in Saudi Arabia: Survey on university women and non-ophthalmic stores. Cont Lens Anterior Eye. 2014; 37 (3): 185-90.

[13] Ibrahim NK, Seraj H, Khan R, et al. Prevalence, habits and outcomes of using contact lenses among medical students. Pakistan Journal of Medical Sciences. 2018; 34: 1429.

[14] Seguí MDM, Cabrero-García J, Crespo A, et al. A reliable and valid questionnaire was developed to measure computer vision syndrome at the workplace. J Clin Epidemiol. 2015; 68 (6): 662-73.

[15] Alabdulkader B. Effect of digital device use during COVID-19 on digital eye strain. Clin Exp Optom. DOI: $10.1080 / 08164622.2021 .1878843$.

[16] The Vision Council. Eyes overexposed: The digital device dilemma: digital eye strain report. [cited 2020 June 29]. Available from: https://visionimpactinstitute.org/wp-content/uploads/2016/03/ 2016EyeStrain_Report_WEB.pdf

[17] Sheppard AL, Wolffsohn JS. Digital eye strain: Prevalence, measurement and amelioration. BMJ Open Ophthalmology. 2018; 3 (1): e000146-e46.

[18] Chiemeke SC, Akhahowa AE, Ajayi OB. Evaluation of Vision-Related Problems amongst Computer Users: A Case Study of University of Benin, Nigeria. World Congress on Engineering. 2007: 2-6.

[19] Ye Z, Abe Y, Kusano Y, et al. The influence of visual display terminal use on the physical and mental conditions of administrative staff in Japan. J Physiol Anthropol. 2007; 26: 69-73.

[20] Logaraj M, Madhupriya V, Hegde SK. Computer vision syndrome and associated factors among medical and engineering students in Chennai. Ann Med Health Sci Res. 2014; 4 (2): 179-85. 
[21] Gonzalez-Meijome JM, Parafita MA, Yebra-Pimentel E, et al. Symptoms in a population of contact lens and noncontact lens wearers under different environmental conditions. Optom Vis Sci. 2007; 84 (4): E296-E302.

[22] Uchino M, Schaumberg DA, Dogru M, et al. Prevalence of dry eye disease among Japanese visual display terminal users. Ophthalmology. 2008; 115 (11): 1982-88.

[23] Kojima T, Ibrahim OMA, Wakamatsu T, et al. The Impact of Contact Lens Wear and Visual Display Terminal Work on Ocular Surface and Tear Functions in Office Workers. Am J Ophthalmol. 2011; 152 (6): 933-40.

[24] Uchino M, Nishiwaki Y, Michikawa T, et al. Prevalence and Risk Factors of Dry Eye Disease in Japan: Koumi Study. Ophthalmology. 2011; 118 (12): 2361-67.

[25] Bali J, Navin N, Thakur BR. Computer vision syndrome: a study of the knowledge, attitudes and practices in Indian ophthalmologists. Indian J Ophthalmol. 2007; 55 (4): 289-94.

[26] Chu C, Rosenfield M, Portello JK, et al. A comparison of symptoms after viewing text on a computer screen and hardcopy. Ophthalmic Physiol Opt. 2011; 31 (1): 29-32.

[27] Blehm C, Vishnu S, Khattak A, et al. Computer vision syndrome: A review. Surv Ophthalmol. 2005; 50 (3): 253-62.

[28] van Setten G, Labetoulle M, Baudouin C, et al. Evidence of seasonality and effects of psychrometry in dry eye disease. Acta Ophthalmol (Copenh). 2016; 94 (5): 499-506.

[29] Maruyama K, Yokoi N, Takamata A, et al. Effect of environmental conditions on tear dynamics in soft contact lens wearers. Invest Ophthalmol Vis Sci. 2004; 45 (8): 2563-8.

[30] Alshamrani AA, Almousa AS, Almulhim AA, et al. Prevalence and Risk Factors of Dry Eye Symptoms in a Saudi Arabian Population. Middle East Afr J Ophthalmol. 2017; 24 (2): $67-73$
[31] Reddy SC, Low CK, Lim YP, et al. Computer vision syndrome: a study of knowledge and practices in university students. Nepal J Ophthalmol. 2013; 5 (2): 161-8.

[32] Schlote T, Kadner G, Freudenthaler N. Marked reduction and distinct patterns of eye blinking in patients with moderately dry eyes during video display terminal use. Graefes Arch Clin Exp Ophthalmol. 2004; 242 (4): 306-12.

[33] Rosenfield M. Computer vision syndrome: a review of ocular causes and potential treatments. Ophthalmic Physiol Opt. 2011; 31 (5): 502-15.

[34] Guillon M, Maissa C, Pouliquen P, Delval L. Effect of povidone $2 \%$ preservative-free eyedrops on contact lens wearers with computer visual syndrome: Pilot study. Eye and Contact Lens. 2004; 30 (1): 34-39.

[35] Acosta MC, Gallar J, Belmonte C. The influence of eye solutions on blinking and ocular comfort at rest and during work at video display terminals. Exp Eye Res. 1999.

[36] García-Ayuso D, Escámez-Torrecilla M, Galindo-Romero C, et al. Influence of the COVID-19 pandemic on contact lens wear in Spain. Cont Lens Anterior Eye. 2020: 1-6.

[37] Morgan PB. Contact lens wear during the COVID-19 pandemic. Cont Lens Anterior Eye. 2020; 43 (3): 213.

[38] Jones L, Walsh K, Willcox M, et al. The COVID-19 pandemic: Important considerations for contact lens practitioners. Cont Lens Anterior Eye. 2020; 43 (3): 196-203.

[39] Bostanci Ceran B, Ozates S. Ocular manifestations of coronavirus disease 2019. Graefes Arch Clin Exp Ophthalmol. 2020: $1-5$.

[40] Seah IYJ, Anderson DE, Kang AEZ, et al. Assessing Viral Shedding and Infectivity of Tears in Coronavirus Disease 2019 (COVID-19) Patients. Ophthalmology. 2020; 2019-21. 